\title{
Particle and pattern discriminant freeze-cleaning method
}

\author{
Kei Hattori, ${ }^{\text {a,b, } * \text { Daisuke Matsushima, }}$, Kensuke Demura, ${ }^{a}$ \\ and Masaya Kamiya ${ }^{a}$ \\ ${ }^{a}$ Shibaura Mechatronics Corporation, Fine Mechatronics Division, Sakae, Yokohama, \\ Kanagawa, Japan \\ ${ }^{b}$ Nagoya University, Center for Low-Temperature Plasma Sciences, \\ National Innovation Complex, Chikusa, Nagoya, Aichi, Japan
}

\begin{abstract}
Background: Although the wet cleaning process has been widely used in semiconductor device manufacturing due to its convenience, it faces theoretical limits. That is, when the size of the objected particle is smaller than $100 \mathrm{~nm}$, it is buried in the stagnant layer where there is substantially no fluid flow.

Aim: Only small particles below the stagnant layer $(<100 \mathrm{~nm})$ is removed without any damage to the fine patterns or substrate: pattern collapse, critical dimension shift, and optical property shift.

Approach: Utilizing unique characteristics of water: volume expansion when freezing, solid (ice) is lighter than liquid (water), and particles adhered the substrate is peeled off from the substrate and rise to the water surface along with the surrounding ice.

Results: By repeating the cycle of cooling, thawing, and rinsing, polystyrene sphere particle of $80 \mathrm{~nm}$ in diameter can be removed with high particle removal efficiency (PRE $>90 \%$ ) and no negative influences on the pattern or substrate.

Conclusions: A new cleaning method for very small $(<100 \mathrm{~nm})$ particles is proposed with high PRE and low damage. This method is thought to be applied to every process if water can infiltrate into the gap between the particles and the substrate.

(C) The Authors. Published by SPIE under a Creative Commons Attribution 4.0 Unported License. Distribution or reproduction of this work in whole or in part requires full attribution of the original publication, including its DOI. [DOI: 10.1117/1.JMM.19.4.044401]
\end{abstract}

Keywords: cleaning; supercooled water; particle removal efficiency; pattern collapse; freeze cleaning; stagnant layer.

Paper 20020 received May 14, 2020; accepted for publication Sep. 17, 2020; published online Oct. 6, 2020.

\section{Introduction}

In recent years, the performance of semiconductor devices has improved owing to improvements such as downscaling, introduction of three-dimensional (3D) structures and new materials, and circuit improvement. ${ }^{1-3}$ For the downscaling process, ArF-immersion (ArF-i) has a resolution limit of $\sim 40 \mathrm{~nm}$, but the minimum critical dimension (CD) of semiconductor devices is already smaller than that in 2012, but the subsequent downscaling of the devices has continued. Extreme ultraviolet (EUV) lithography was recently introduced with a resolution limit of $\sim 13 \mathrm{~nm}$ for single patterning. Currently, the minimum size of semiconductor device is thought to be $\sim 7 \mathrm{~nm}$, so even if the EUV lithography technology is used, the minimum CD of the semiconductor devices is smaller than its limit. Downscaling can be realized using patterning techniques such as pattern splitting and self-aligned patterning on a wafer. ${ }^{4,5}$ Although requirements for a

*Address all correspondence to Kei Hattori, E-mail: kei.hattori@shibaura.co.jp 
decrease in the photomask dimensions are reduced, fine patterning and cleaning processes exhibit significant performance demands.

Here, we confirm why not only fine pattering technology but also cleaning technology is important to realize the cutting edge devices. Semiconductor device manufacturing is basically consisted of repetition of thin-film processes. That is, by forming a desired thin film is deposited on the substrate, transferring the circuit pattern to the photoresist (mask material) through a photomask (master plate), and processing underlayered film according to this mask, then the desired material is created as a fine pattern. If before and/or after fine patterning, there is foreign material on the circuit area, it leads a circuit open or short. This means during these processes, substrate should be kept clean or all the foreign materials should be removed from the substrate. So before and after each process, cleaning process is inserted multiple times. In addition, low (none)-damage cleaning process is strongly required because of multiple use. That is why the cleaning technology is so important to realize the cutting edge devices. In photomask fabrication, basically this thin-film process concept is introduced. So in the photomask fabrication line, cleaning processes are used multiple times (timing and frequency depend on the production line performance and requirement to the mask performance). Thus, the efficient and low-damage cleaning process is required.

So photomask cleaning process is applied multiple times in its production and also used in high-volume manufacturing.

Furthermore, differences exist between the causes of the significant performance demands for the mask and wafer processes. Given that a mask is a master plate used in the mass production of semiconductor devices, it should not contain defects (this corresponds to $100 \%$ yield). So a repair process is also introduced to the mask fabrication. But to reduce the initial number to repair, that is, to fabricate the complete mask, cleaning steps are introduced multiple times in the mask production flow much often than that of wafer process.

Additionally, a mask is repeatedly used in the same number of processes as the number of manufactured devices in the high-volume mass production of semiconductor devices. Owing to repeated use, a mask is soiled by defects, such as growing defects. To remove these defects, the mask must be cleaned at a specific frequency.

Next, we discuss a target size of the particles to be cleaned. For an optical photomask, the minimum diameter of the particle that requires cleaning is $\sim 100 \mathrm{~nm}$ considering that the $\mathrm{CD}$ of a photomask is four times larger than that of wafer and $\leq 50 \mathrm{~nm}$ for EUV. In nanoimprint lithography (NIL), ${ }^{6,7}$ a fine pattern is directly translated into a template without reduction; thus, the target minimum particle diameter is $\sim 10 \mathrm{~nm}$. However, particles with diameters smaller than $100 \mathrm{~nm}$ are hard to remove, as described below.

Various processes (e.g., dry cleaning) with respect to cleaning have been investigated. ${ }^{8-10}$ Cleaning technologies based on liquids have also been employed given that physical forces are effective in particle removal. Accordingly, their use is expected to increase over time. However, the particle removal efficiency (PRE) of wet cleaning technologies significantly decreases for the removal of particles with diameters smaller than $100 \mathrm{~nm}$. In a wet cleaning process, the fluid drag force $F_{d}$, which is the particle removal force is given by ${ }^{11}$

$$
F_{d} \propto d_{p}^{2} u^{2}
$$

where $d_{p}$ is the particle diameter and $u$ is the velocity of a moving fluid. The main adhesion force of particles to a substrate is the Van der Waals adhesion force $\left(F_{\mathrm{VdW}}\right)$, which is proportional to $d_{p}{ }^{11}$ Given that the force required to remove the particle from the substrate is quadratically proportional to the particle diameter and the particle adhesion force on the substrate is linearly proportional to the particle diameter, hence, the adhesion force significantly increases in accordance with a decrease in the particle size. In addition, a layer referred to as the stagnant layer in the immediate proximity of the substrate has almost no fluid flow. The layer thickness is $\sim 100 \mathrm{~nm}$. According to hydrodynamics, the velocity of a Newtonian fluid such as water is zero at a fixed surface, and the velocity increases in accordance with an increase in its distance from the surface. A stagnant layer is a region where the liquid velocity is significantly low. Thus, it is considered as the zero region and is $\sim 100 \mathrm{~nm}$ in the case of water. 
Therefore, when the particle diameter is smaller than $\sim 100 \mathrm{~nm}$, almost no effective particle removal force exists, resulting in a significantly poor cleaning performance. From the abovementioned consideration, it is thought to be a theoretical limit to remove a smaller than $~ 100-\mathrm{nm}$ particle. Tomita ${ }^{12}$ investigated the decrease in the stagnant layer thickness by controlling the liquid viscosity and the surface energy of the solid interface. The stagnant layer thickness can be decreased to an extent, and particles with diameters of $40 \mathrm{~nm}$ can be removed with a high PRE by increasing physical impact. However, this increases the number of fine pattern defects; thus, an increase in the physical impact (cleaning ability) results in a tradeoff between the PRE and pattern defects.

General cleaning process utilized physical and/or chemical action to the entire substrate for cleaning, so the fine patterns and the particles seem the same object. Therefore, as the applicable cleaning condition, it is necessary to find a condition that the patterns are not peeled off from the substrate and only the particles are peeled off. The smaller the pattern size is, the weaker the adhesion force is, and then the difference between the adhesion force of the pattern to the substrate and that of the particle is becoming narrower. This is the reason why cleaning becomes difficult with the downscaling.

Moreover, the freeze-cleaning equipment used in this study was designed and manufactured with a focus on the following phenomena: (1) the expansion of water during its phase transition to ice, (2) the instantaneous partial freezing of a portion of supercooled water upon its induced phase transition to ice, and (3) the ice growth by continuous cooling.

In Sec. 2, we discuss these phenomena in detail. Section 2.1 describes the fundamental characteristics of water and supercooled water. It also describes what happens when water freezes. Section 2.2 describes the motion of the particle in the water. By solving the equation of motion, we estimate the velocity and the time to travel of $100 \mathrm{~nm}$ (to escape from the stagnant layer) of particles in the water. And Sec. 2.3 describes the application of these phenomena to freeze cleaning with a model proposal.

\section{Characteristics of Water and the Freeze-Cleaning Mechanism}

\subsection{Characteristics of Water and Supercooled Water}

This section presents a discussion on the freezing process of supercooled water and its physical properties with respect to literature. The freezing point of water is $0^{\circ} \mathrm{C}$; however, it can remain liquid at significantly lower temperatures under a given set of conditions with the lowest reported temperatures between $-40^{\circ} \mathrm{C}$ and $-48^{\circ} \mathrm{C} .{ }^{13,14}$ Moreover, triggers, such as flow, vibration, fine patterns, and particles, cause water freezing and ice formation. The freezing temperature for the supercooled state varies depending on the conditions. However, the entire system rapidly freezes upon the induction of freezing. When freezing is triggered, the temperature of the water and ice is $0^{\circ} \mathrm{C}$, regardless of the supercooled water temperature. Consider the case of a cooled substance and the phase transition from liquid to solid. First, the liquid is cooled, which lowers the internal energy by lowering the temperature. When the freezing temperature is reached, this temperature is maintained. Instead, in the phase transition, solidification begins. In this study, water is cooled to a temperature exceeding its inherent solidification temperature to become supercooled water. The water phase transitions to ice when a stimulus is provided, and proceeds as follows. First, accumulated heat (solidification heat in this case) is used as the latent heat. The amount of accumulated heat depends on the temperature when the supercooled state finishes. The accumulated heat should be used as a solidification heat all at once. Therefore, the entire system freezes like a sherbet, and the temperature goes up to the freezing temperature. If water is cooled very slowly, it freezes at $0^{\circ} \mathrm{C}$ and maintains this temperature until all the water is frozen. The amount of ice formed from the initial amount of water can be estimated. According to Yokoyama, ${ }^{14}$ the physical change is an adiabatic process, and a quantity of heat is emitted until the water at $0^{\circ} \mathrm{C}$ reaches the point at which the final temperature of the supercooled state is equal to the quantity of heat emitted during freezing for ice formation. For example, $19 \%$ and $52 \%$ of the total water freeze if the final temperature of the supercooled state is $-15^{\circ} \mathrm{C}$ and $-38^{\circ} \mathrm{C}$, respectively. Moreover, the ice expansion speed is $\sim 25 \mathrm{~cm} / \mathrm{s}$ when the final temperature of the supercooled state is $-15^{\circ} \mathrm{C} .{ }^{14}$ 


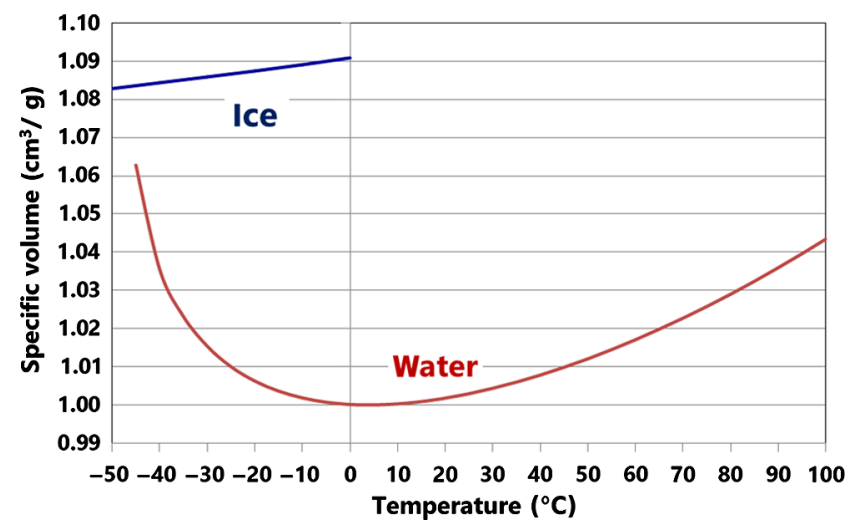

Fig. 1 Temperature dependence of the specific volume of ice and water plotted based on Refs. 15 and 16.

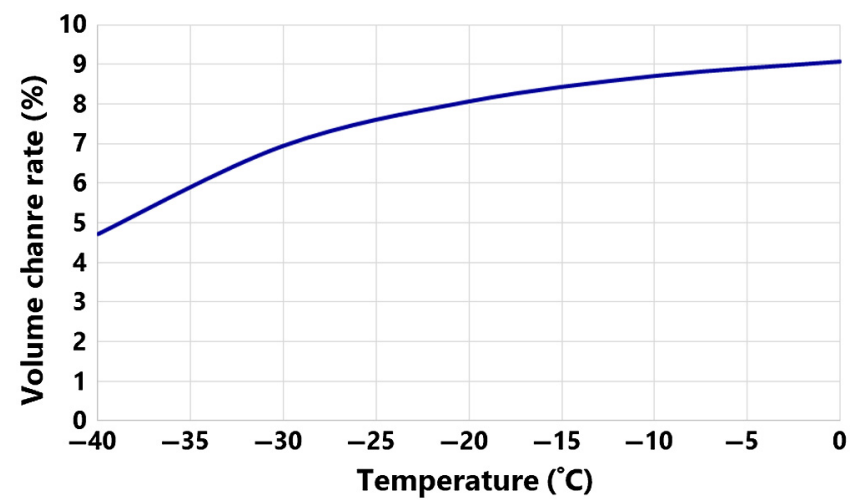

Fig. 2 Volume change rate when supercooled water is frozen.

The specific volume, which is the inverse of the water density, is the lowest at $4^{\circ} \mathrm{C}$ and increases at temperatures above and below this threshold. Similarly, the specific volume of ice decreases in accordance with a decrease in temperature below $0^{\circ} \mathrm{C}$. Figure 1 shows the temperature dependence of the specific volume of ice and water between $-50^{\circ} \mathrm{C}$ and $100^{\circ} \mathrm{C} .{ }^{15,16}$ Water below $0^{\circ} \mathrm{C}$ is supercooled water; however, the specific volume gradually changes as the water changes from normal to the supercooled state (Fig. 1). The volume change between $0^{\circ} \mathrm{C}$ and $-38^{\circ} \mathrm{C}$ is $\sim 3.6 \%$ and $\sim 0.6 \%$ for water and ice, respectively.

Figure 2 shows the volume change estimated from the above-mentioned values based on the assumption that supercooled water transitions into ice at $0^{\circ} \mathrm{C}$. Moreover, with respect to this supercooled water, the volume increases by $4.7 \%$ to $9.1 \%$ when compared to that immediately prior to freezing. Thus, the volume change causes the particles to be removed from the substrate. Although the volume change decreases in accordance with the decrease in temperature of the supercooled water, a volume change of over $4 \%$ is maintained, this is considered as sufficient for particle removal.

\subsection{Motion of Particles, Ice, and Particles in the Water}

According to the above-mentioned consideration, the particle adsorbed on the substrate is thought to be removed from the surface if surrounded by the ice. Next, we consider a motion of the particle surrounded by the ice [freeze cleaning (FC) particle]. To simplify the consideration, all of the particles are assumed as sphere and temperature is $0^{\circ} \mathrm{C}$. If the $\mathrm{FC}$ particle is very small, the motion is assumed as a laminar flow. In this case, the forces that act on the FC particle are assumed as buoyancy $\left(F_{b}\right)$, gravity $\left(F_{g}\right)$, and the drag force $\left(F_{d}\right)$ described in Fig. 3. Buoyancy is given by 


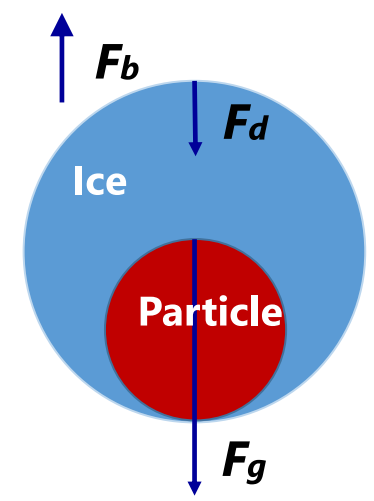

Fig. 3 Forces act on the particle surrounded with ice.

$$
F_{b}=\rho_{w} V_{\mathrm{FC}} g,
$$

where $\rho_{w}$ is the density of water (not that of the FC particle) and $V_{\mathrm{FC}}$ is volume of the FC particle.

Radii, density, volume, and mass of the ice, the particle, and the FC particle are described as: $r_{\text {ice }}, r_{p}, r_{\mathrm{FC}}, \rho_{\text {ice }}, \rho_{p}, \rho_{\mathrm{FC}}, V_{\mathrm{ice}}, V_{p}, V_{\mathrm{FC}}$, and $m_{\mathrm{ice}}, m_{p}, m_{\mathrm{FC}}$, respectively. Drag force is given by ${ }^{17}$

$$
F_{d}=6 \pi \mu r_{\mathrm{FC}} v_{\mathrm{FC}}
$$

where $\mu$ is the viscosity of water and $v_{\mathrm{FC}}$ is the velocity of the FC particle.

Then the equation of the motion is given by

$$
m_{\mathrm{FC}} \frac{\mathrm{d} v_{F C}}{\mathrm{~d} t}=\left(\rho_{w} V_{\mathrm{FC}}-m_{\mathrm{FC}}\right) g-6 \pi \mu r_{\mathrm{FC}} v_{\mathrm{FC}} \text {. }
$$

Solving Eq. (4) by taking the origin at the surface of the substrate, and the $x$ axis as the up-forward vertical direction,

$$
v_{\mathrm{FC}}=\frac{m_{b} g}{k}\left(1-e^{-\frac{k}{m_{b}} t}\right),
$$

and

$$
x_{\mathrm{FC}}=\frac{m_{b} g}{k}\left(t+\frac{m_{b}}{k} e^{-\frac{k}{m_{b}} t}\right) \text {, }
$$

where

$$
\begin{gathered}
m_{b}=\rho_{w} V_{\mathrm{FC}}-m_{\mathrm{FC}}, \\
k=6 \pi \mu r_{\mathrm{FC}} .
\end{gathered}
$$

When $t \rightarrow \infty$ in Eqs. (5) and (6)

$$
\begin{gathered}
v_{\mathrm{FC} \infty} \rightarrow \frac{m_{b} g}{k}, \\
x_{\mathrm{FC} \infty} \rightarrow \frac{m_{b} g}{k} t=v_{\mathrm{FC}} t
\end{gathered}
$$

These results mean that the FC particle motion is as follows: FC particle starts by buoyancy at the beginning of its motion and slowed down by the drag force of proportion to the velocity, and after buoyancy and drag force are balanced it reach to the terminal velocity Eq. (9).

In accordance with the above consideration, we estimate the actual value of our experiment: detailed condition for the experiment will be mentioned later. At the first, we estimate the 
condition of buoyancy is strongest. Particle is polystyrene of $80 \mathrm{~nm}$ in diameter and density $\rho_{p}=1.05 \mathrm{~g} / \mathrm{cm}^{3}$. At $0^{\circ} \mathrm{C}, \rho_{w}=1 \mathrm{~g} / \mathrm{cm}^{3}$ and $\rho_{\text {ice }}=0.917 \mathrm{~g} / \mathrm{cm}^{3}$.

$$
V_{\text {ice }} \geq 0.62 V_{p} .
$$

In the case of $V_{\text {ice }}=V_{p}$, terminal velocity of FC particle $\left(v_{T 80 \mathrm{~nm}}\right)$ is estimated with Eq. (9) and $\mu=1.79 \times 10^{-3} \mathrm{~Pa} \cdot \mathrm{s}$ at $0^{\circ} \mathrm{C},{ }^{18}$

$$
v_{T 80 \mathrm{~nm}} \cong 5.1 \times 10^{-11} \mathrm{~m} / \mathrm{s} .
$$

And in this case, time constant in Eqs. (5) and (6) is estimated as

$$
\frac{1}{\frac{k}{m_{b}}}=\frac{v_{T 80 \mathrm{~nm}}}{g} \cong 5.2 \times 10^{-12} / \mathrm{s}
$$

This estimation means that the FC particle achieves to the terminal velocity immediately after the float motion begins, and its speed is extremely low. Therefore, the FC particle will be adrift very close to the substrate surface and never rise up beyond the stagnant layer. This means that the particle is not removed at all.

In Sec. 2.3, we will further consider the water/ice behavior on the substrate and propose a brand new cleaning process.

\subsection{Model of Freeze Cleaning}

Now we further consider the water/ice behavior and propose a cleaning process with a model. From now on, the consideration is along with our experimental condition mentioned later: water is poured on the substrate with thickness of $60 \mu \mathrm{m}$, cooled at the bottom via the substrate, and water is frozen at $-38^{\circ} \mathrm{C}$ through supercooled water. And after frozen, the ice is continuously cooled during the thaw process. After the entire water on the substrate phase change to the ice, it is thawed by rinsing with $20^{\circ} \mathrm{C}$ water, and then the next step is started by pouring $60-\mu \mathrm{m}$-thick water again after spinning off the water thickness to about $10 \mu \mathrm{m}$. On the substrate surface, a fine pattern is fabricated, a particle is adsorbed.

In accordance with Fig. 4, we discuss the water/ice behavior. Figure 4(a) describes the water from $4^{\circ} \mathrm{C}$ to $-38^{\circ} \mathrm{C}$ before freeze begins. In this temperature region, cooler water is lighter than warmer one. So cooler water rises up to the surface, and warmer water sinks down to the bottom. Therefore, a convection occurs: this is opposite direction of heating, and specific volume of supercooled water at $-38^{\circ} \mathrm{C}$ is almost the same value of $80^{\circ} \mathrm{C}$ water (see Fig. 1). This water motion is thought to lead a local ununiformity of density, flow, and wave of the water. So this ununiformity of the physical properties may be a trigger point of freeze. As mentioned in Sec. 2.1, when the temperature of the water reaches to $-38^{\circ} \mathrm{C}$, about $52 \%$ of the volume of the water is frozen [Fig. 4(b)]. In Fig. 4(b), surrounding water of particle 1 is frozen but that of particle 2 is not frozen, because the ice formation is thought to be determined stochastically. In the case of the particle 1, between the substrate and the particle there is not enough space to expand the volume when the water freezes. Therefore, the ice pushes the particle aside to make the space for expansion [red arrows in Fig. 4(b)]. The other portion on particle 1, the ice surrounding the particle does not feel any reaction force and merely expands in volume when freezing [we ignore a weak compression force because it does not affect to the particle motion; blue arrows in Fig. 4(b)]. At the fine patterns, if between patterns and the substrate there are no gaps that water infiltrate to, no force acts to remove them [blue arrows in Fig. 4(b)]. At the ice created in water due to some fluctuation of physical properties (density ununiformity, wave, and flow), the volume increases by about 5\% when compared to that immediately prior to freezing (Sec. 2.1) and no force acts to remove anything. Blue arrows are shown at one of the ice to avoid complication in Fig. 4(b). These phenomena are considered to be one of the key factors in forming the removal force of the particle from the substrate. During continuous cooling until the entire water change to the ice, the temperature of the liquid-solid coexistence state is kept at $0^{\circ} \mathrm{C}$. So, macroscopically, the heat (minus amount) should be used as a solidification heat on a 


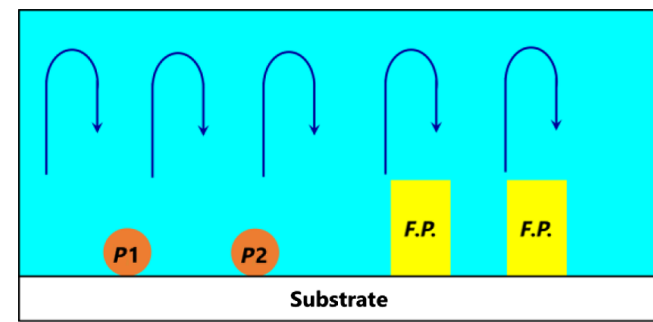

(a)

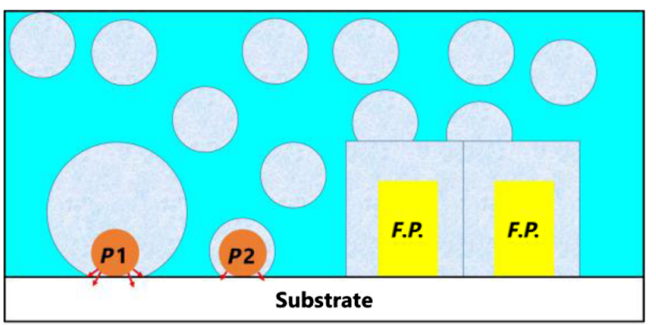

(c)

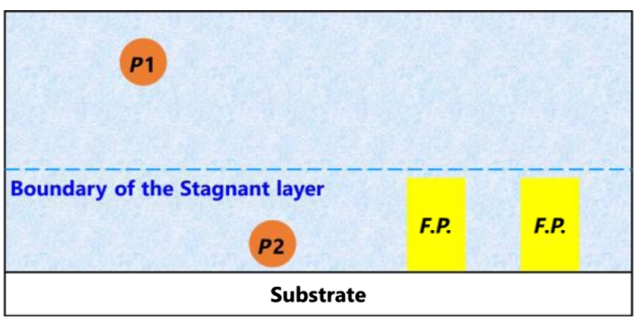

(e)

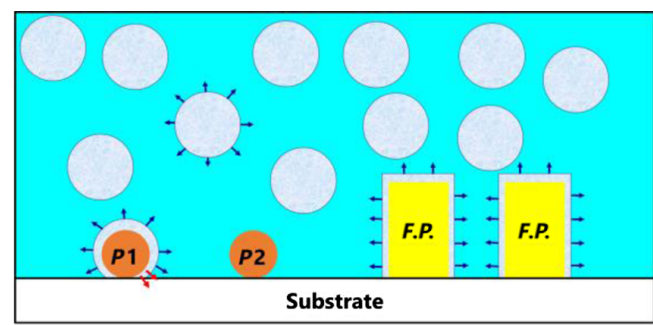

(b)

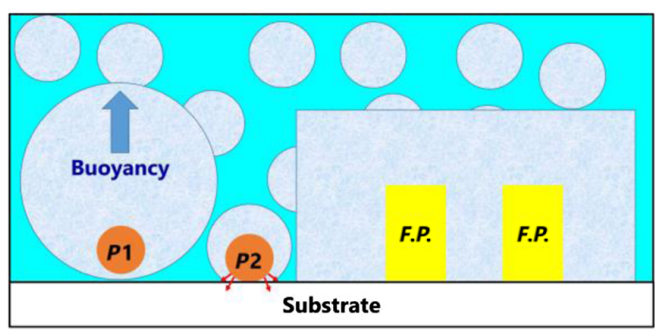

(d)

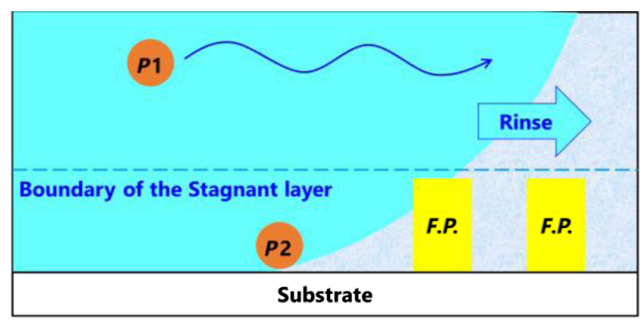

(f)

Fig. 4 The model of freeze-cleaning method. (a) Water behavior from $4^{\circ} \mathrm{C}$ to before freezing begins $\left(-38^{\circ} \mathrm{C}\right)$, (b) ice formation in the water and particle removal force by volumetric expansion, (c) ice growth by continuously cooling from the substrate, (d) escape the FC particle by buoyancy from the substrate, $(e)$ entire water is frozen, and (f) particle only escaped from the stagnant layer is rinsed away from the substrate.

priority basis. No temperature gradient exists inside the sherbet such as water, no heat shift occurs. The heat exchange occurs at the water surface and the bottom of the water. So the ice created inside the water is thought to be adrift in the water and the size is not change. At the bottom of the water, fine patterns surrounded by the ice, particles surrounded by the ice and the water contact to the substrate. In this part, the ice is thought to be grown by cooling from the substrate and removal force acts at the bottom like as Fig. 4(b). And also it is thought that the ice creation may occur at the particle that was not triggered prior timing. This image is shown in Fig. 4(c). At the water surface, water may be heated up by the air microscopically, but the heat from the air mainly should be used as a heat of fusion of the ice at/adjacent to the water surface, and the bulk temperature is kept at $0^{\circ} \mathrm{C}$. And then the particle surrounded large ice rises to water surface [Fig. 4(d)]. Finally, the entire water phase change to the ice, and some of the particles escaped from the stagnant layer and the other particles are still remain on the substrate surface [Fig. 4(e)]. And then the ice is thawed by rinsing with $20^{\circ} \mathrm{C}$ water [Fig. 4(f)].

The water infiltration into gaps depending on the gap size is briefly described below. When a pattern pitch is within the millimeter to micrometer scales, the "lotus effect" is predominant, which does not result in water infiltration into the pattern gap. However, when the pattern pitch is at the submicrometer scale, the surface tension, which is primarily the capillary force, is predominant. As reported, water infiltrates the gaps between small patterns via gas dissolution. ${ }^{19-21}$ The tool and the process developed herein have been used by Tanabe et al. ${ }^{22}$ to remove particles stuck between small gaps $(\sim 80 \mathrm{~nm})$. A further investigation is required on water infiltration into all gaps with respect to the materials, shape, and surface energy. This paper presents a discussion on water infiltration into the small gaps between the substrate and the particle. 
Now, we estimate how large ice is needed to escape from the stagnant layer easily according to the discussion in Sec. 2.1.

First, we estimate when the particle weight can be ignored. The weight of the FC particle is given as

$$
m_{\mathrm{FC}}=\rho_{\mathrm{FC}} V_{\mathrm{FC}}=y \rho_{\text {ice }} V_{\text {ice }}+(1-y) \rho_{p} V_{p},
$$

where $y$ is the proportion of the ice in FC particle. We assumed when the second term on the right side is smaller than 1/100 compare to the first term on the right side, the particle weight can be ignored. In the case where the particle is polystyrene $\left(\rho_{p}=1.05 \mathrm{~g} / \mathrm{cm}^{3}\right)$ of $80 \mathrm{~nm}$ in diameter, $r_{\text {ice }}$ is estimated as

$$
r_{\text {ice }} \cong 194 \mathrm{~nm} \text {. }
$$

And terminal velocity $v_{T 389}$ is estimated by Eq. (9)

$$
v_{T 389} \cong 3.9 \times 10^{-10} \mathrm{~m} / \mathrm{s} \text {. }
$$

This velocity is thought to be still too low to escape from the stagnant layer. In the case of terminal velocity to reach faster than $1 \mu \mathrm{m} / \mathrm{s}$, the ice radius needs larger than $3.1 \mu \mathrm{m}$. When the ice $\left(\approx \mathrm{FC}\right.$ particle) radius is $3.5 \mu \mathrm{m}$, terminal velocity $v_{T 3500}$ and time to travel of $100 \mathrm{~nm} t_{100 \mathrm{~nm}}$ are estimated as

$$
v_{T 3500}=1.2 \mu \mathrm{m} / \mathrm{s}
$$

and

$$
x_{100 \mathrm{~nm}} \cong 80 \mathrm{~ms} \text {. }
$$

In our experiment mentioned later, liquid-solid coexistence time is about $5 \mathrm{~s}$. So $80 \mathrm{~ms}$ is short enough that FC particle escapes from the stagnant layer.

This study involved design, manufacture, and evaluation of the equipment to realize the above-mentioned states, namely, the freezing phenomenon of the supercooled water (e.g., particle-induced freezing), the volumetric expansion at freezing used to remove the particle from the substrate surface, and the ice growth of the FC particle to escape from the stagnant layer by buoyancy.

The following can be realized considering the utilization of changes in the physical properties during the freezing of supercooled water (i.e., the volumetric expansion is the force utilized for the particle removal from the substrate surface. And ice growth utilized for giving the enough velocity to escape from the stagnant layer).

Upon pouring and cooling of water on the substrate, freezing occurs via the supercooled phase. Several possible stimuli, such as water flow, vibration, particles, and fine patterns, can be employed. Freezing may be induced by particles, fine patterns, and some fluctuation of physical properties [Figs. 4(a) and 4(b)]. The cooled water rises to the water surface owing to its lower mass at a lower temperature. Thus, water cooling is preferably performed from the substrate side. If the water is cooled from its surface, the cooled water is retained at the surface by its specific weight (Fig. 1); thus, the cooling of the substrate surface, which is the bottom of the water, is only because of the heat conductivity. This indicates a decrease in the cooling efficiency of the substrate surface. In addition, it is highly probable that freezing is induced at lower temperatures, thereby starting at the water surface and not at the particle. This case stimulus is thought to be a fluctuation of the physical property at the water surface and not the particle nor the fine pattern but the wave. Upon the particle-induced freezing of the supercooled water, after the particle removal from the substrate, it rises to the water surface due to the buoyancy of the surrounding ice, which is lighter than the supercooled water (Fig. 1). With the continuous cooling of water, the ice size increases and so does the buoyancy. The particles that rise above the stagnant layer are rinsed from the substrate at the subsequent rinse step. Owing to the insufficient amount of particles removed by one process cycle, the process is repeated. Small particles measuring $80 \mathrm{~nm}$ are removed with a PRE larger than $95 \%$. 


\section{Materials and Methods}

\subsection{Cleaning Sample and Particle Measuring Method}

The samples for this study were prepared as follows: commercially available 6025 quartz substrate was coated with a polystyrene latex (PSL) aqueous solution (Thermo Scientific, 3080A model) having particle diameters of $80 \mathrm{~nm}$ under optimum conditions. The number of PSL particles was adjusted to $10,000 \pm 10 \%$. The number of particles on the substrate was measured using a mask substrate/blank inspection tool (Lasertec, Magics M2350: measurement limit is $80 \mathrm{~nm}$ or larger). For the evaluation of the collapse of the fine patterns, the evaluation samples and data were obtained from Kioxia Co., Ltd., and a joint research was conducted. ${ }^{22}$ Evaluated sample was MoSi alloy-based attenuated phase shift material of 60-nm height, isolated pattern with minimum size of $50 \mathrm{~nm} \times 75 \mathrm{~nm}$ was used.

The PRE is used as an indicator of the particle cleaning efficiency that is obtained using Eq. (19) as follows:

$$
\operatorname{PRE}(\%)=\frac{N_{\text {initial }}-N_{\text {final }}}{N_{\text {initial }}} \times 100
$$

where $N_{\text {initial }}$ represents the number of particles on the substrate measured using a particle counter prior to the cleaning process, and $N_{\text {final }}$ represents the number of particles after the process.

\subsection{Cleaning Tool Configuration}

The freeze-cleaning module used in this study was developed as a critical module of a novel single photomask cleaning system ${ }^{23}$ (Shibaura, MC150-plus). Figure 5 shows an overview of the cleaning system MC150-plus, which can be employed for the flexible setup of MC150-plus various module types. The system employed in this study consisted of two standard mechanical interface pods, a double transfer arm (upper: dry; lower: wet), an ultraviolet (UV) irradiation module, a flip hand module, a spin module, and a freezing module. Figure 6 shows an overview

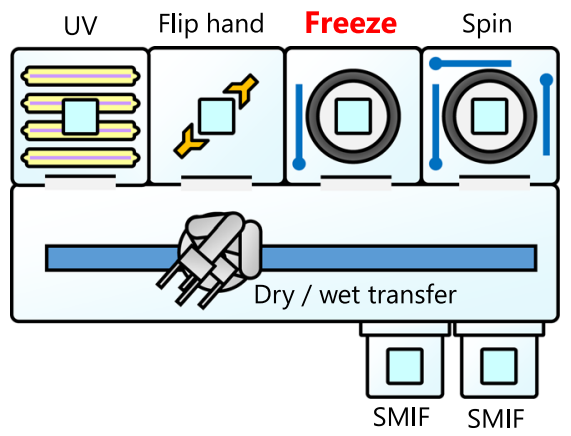

Fig. 5 Outline of MC150-plus and layout of the freeze-cleaning module.

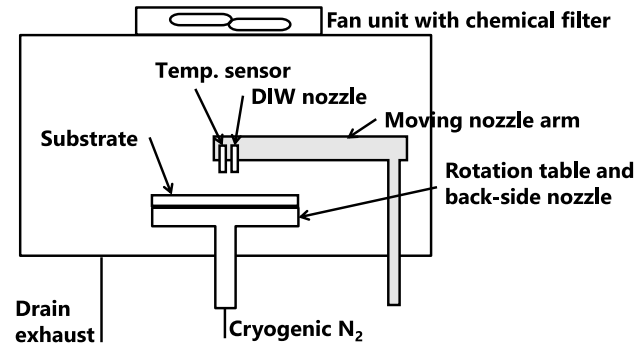

Fig. 6 An overview of the freeze-cleaning module. 
of the freeze-cleaning module. The chamber was equipped with a rotating mechanism to hold and rotate a mask, a backside nozzle to eject cryogenic $\mathrm{N}_{2}$ into the underside of the mask, and a moving nozzle arm to dispense deionized water (DIW) onto the upper-side surface of the mask. The ceiling of the chamber had a fan unit with a chemical filter. The inside of the chamber was kept clean. In addition, the moving nozzle arm was equipped with a radiation thermometer (Micro-Epsilon, CTF-SF15-C3) to measure the surface temperatures of the DIW and the substrate.

\subsection{Cleaning Procedure}

The substrate was processed based on UV irradiation in a UV module $\left(70 \mathrm{~mW} / \mathrm{cm}^{2}, 4 \mathrm{~min}\right)$, precooling (freezing, thawing, rinsing) $\times n$-cycles, and spin drying.

First, the precooling step duration was fixed at $600 \mathrm{~s}$ through a preliminary check of the substrate cooled to $-70^{\circ} \mathrm{C}$ and saturated. During precooling, DIW was continuously supplied to prevent the water vapor from accumulating on the substrate. When the precooling was completed, the water supply was stopped under predetermined conditions to maintain an appropriate amount of water on the substrate (about $60-\mu \mathrm{m}$-thick water was remained). The surface temperature was monitored using the radiation thermometer. The substrate was cooled and frozen using cryogenic $\mathrm{N}_{2}$ gas. Thereafter, the substrate was thawed by rinsing with water at room temperature (i.e., $\sim 17^{\circ} \mathrm{C}$ in this study). Between cycle and cycle, the substrate surface was kept wet (water thickness was maintained thicker than about $10 \mu \mathrm{m}$ during rinsing). After the substrate was subjected to multiple freeze-thaw cycles, it was rinsed using DIW and then spin-dried. During the above-mentioned treatment, with the exception of the final drying step, cryogenic $\mathrm{N}_{2}$ gas was continuously supplied.

\subsection{Temperature of Substrate Surface with Respect to the Start Time of the Thawing Step}

In accordance with the above discussion, we set the experiment procedure, but it seems a little long process time is required. Especially, it takes a considerable amount of time to thaw from the entire substrate surface if frozen [Fig. 4(f)]. On the other hand, if rinsing is started in the liquidsolid coexistence state, the amount of ice adhering to the substrate is thought to be small [Fig. 4(d)]. So it can be expected that the time required for thawing can be shortened. So we added an experiment based on this consideration.

Figure 7 shows the temperature of the substrate surface throughout the process as monitored using the radiation thermometer. Given that the sensitivity of the radiation thermometer at low temperatures ranged from 8 to $14 \mu \mathrm{m}$, the measured temperature was that of the outermost surface, as the infrared (IR) light was absorbed by water with a large absorbance. The temperature trend at each step is presented as follows [Fig. 7(a)]:

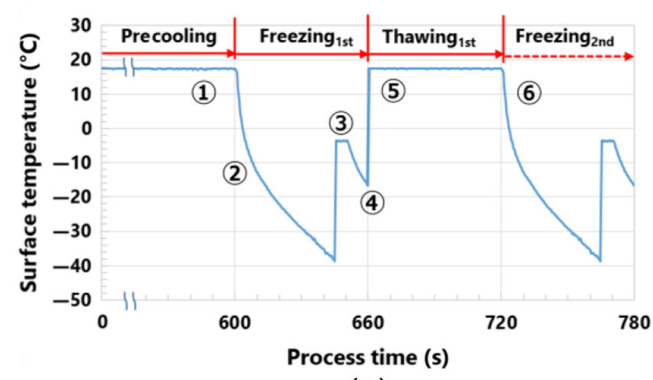

(a)

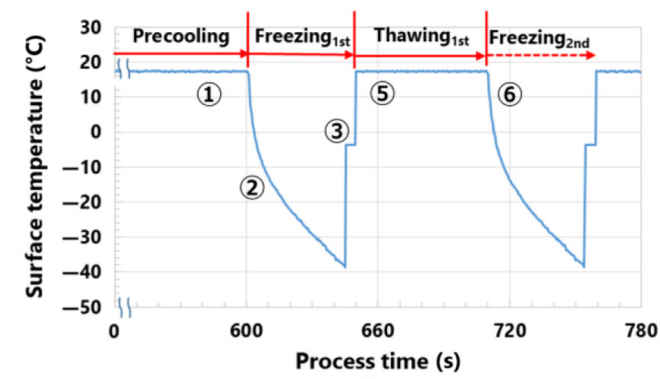

(b)

Fig. 7 Substrate surface temperature with respect to the process using cryogenic $\mathrm{N}_{2}$ on the underside of the substrate. (a) The thawing step begins after the temperature decreases for the second time in the freezing step. This corresponds to Fig. 4(f). (b) The thawing step begins when the temperature is maintained at $-4^{\circ} \mathrm{C}$ in the freezing step. This corresponds to Fig. $4(\mathrm{~d})$. 
1. The surface temperature of the rinse water during precooling was $\sim 17^{\circ} \mathrm{C}$.

2. Water was cooled using cryogenic $\mathrm{N}_{2}$ gas via the substrate, passed through the freezing temperature of $0^{\circ} \mathrm{C}$, and froze at $\sim-38^{\circ} \mathrm{C}$ via the supercooling phase. Process time count starts at the beginning this step.

3. As expected, the temperature increased to $0^{\circ} \mathrm{C}$. However, the cooling speed is significantly high in our system, so the actual temperature may be balanced at slightly lower than $0^{\circ} \mathrm{C}$ $\left(\sim-4^{\circ} \mathrm{C}\right)$, which resulted in a liquid-solid coexistence phase. Moreover, continuous cooling increased the ice size. However, the temperature was maintained.

4. The temperature decreased again after all the water underwent a phase transition into ice.

5. Rinse water was poured onto the substrate surface to induce thawing: this step corresponds to Fig. 4(f), and the process time count ends at the beginning of this step. All of the ice melted and was rinsed from the substrate, constituting the final step of one process cycle.

6. The rinse water supply was stopped, resulting in a decrease in excess water and inducing freezing as the start of the subsequent cycle.

Figure 7(b) shows that steps 1 to 3 are the same as Fig. 7(a). Thawing began at step 3, where the temperature was maintained at $\sim-4^{\circ} \mathrm{C}$ : this step corresponds to Fig. 4(d). Steps 5 and 6 were the same as Fig. 7(a).

\section{Results and Discussion}

\subsection{PRE versus Repeat Cycle Number}

A result processed in accordance with Fig. 7(a) was obtained with PRE $=83 \%$. In this case, process time was $90 \mathrm{~s}$.

Figure 8 shows the PRE dependence on the number of cycles. The procedure was performed by referring to Fig. 7(b). In this case, process time was $47 \mathrm{~s}$. The PRE was 52\% when 10 cycles of the process were conducted, 95\% after 30 cycles, and $94 \%$ after 60 cycles. Figure 8 also shows the particle map after each cycle. The PRE uniformly increased in accordance with an increase in the number of repetitions and then saturated at 30 cycles.

As expected, the supercooled water thawing on the substrate surface by the formation of a liquid-solid phase removed the particles from the substrate. Moreover, with an increase in the number of cycles, a PRE $>90 \%$ can be achieved.

$\mathrm{PRE}_{n}$ is given in Eq. (20) when the number of particles is sufficient:

$$
\mathrm{PRE}_{n}=1-\theta^{n},
$$

where $\theta$ is the remaining probability after one cycle of the cleaning process. A particle count greater than $\sim 5000$ is sufficient. As shown in Fig. 8, $\theta$ was estimated when the cycle number was 10 and the PRE $=52 \%$ :

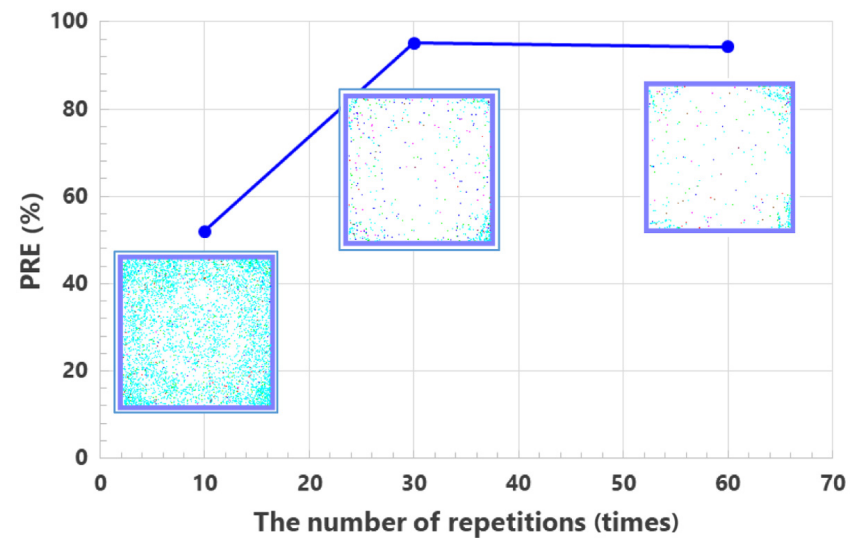

Fig. 8 PRE with respect to the number of cycles. 


$$
\theta=93 \%
$$

and

$$
\mathrm{PRE}_{1}=7 \% \text {. }
$$

With references to the values above and the literature, ${ }^{14} \sim 52 \%$ of the total DIW transitioned into ice at the end of the supercooling phase at $-38^{\circ} \mathrm{C}$. Thus, $\sim 13 \%$ of the phase change contributes to the particle removal. The estimated value of $\theta$ is not accurate. However, the amount of frozen supercooled water that contributes to the particle removal can be estimated.

\subsection{PRE versus Freezing Step Time}

Figure 9 shows the PRE dependence on the freezing time. In this case, the procedure was done by referring to Figs. 7(a) and 7(b). The number of cycles was 10. The PRE was 52\% at $47 \mathrm{~s}$ in the liquid-solid phase, $83 \%$ at $90 \mathrm{~s}$ in the solid phase, and $99 \%$ at $600 \mathrm{~s}$ in the solid phase. In addition, the ice was crushed at $600 \mathrm{~s}$.

Hence, the PRE for the solid phase thawing improved by $8 \%$, which was slightly higher compared to that in the liquid-solid phase thawing. The reason why this difference is considered is as follows: in accordance with the discussion above, rinse starts at the timing of Fig. 4(d), the amount of the particles captured and removed away from the stagnant layer by the ice should be halfway. On the other hand, rinse starts at the timing of Fig. 4(f), all of the particle is captured and removed away from the stagnant layer. And also, the difference in the thermal shrinkage between the substrate and the ice may act as a shear force on the particles (linear thermal expansion coefficient of quartz and ice is $3.4 \times 10^{-7}$ and $5.4 \times 10^{-7}$, respectively). However, at $600 \mathrm{~s}$, a significant improvement of $99 \%$ was observed in the PRE. This can be attributed to the ice shrinkage by continuous cooling, followed by crushing with buckling and removal from the substrate. However, the particles and the fine patterns may also be removed from the substrate; thus, pattern collapse may occur.

\subsection{Pattern Collapse and PRE}

A pattern collapse may occur when the stress applied to the pattern surface is sufficient for crushing ice. Therefore, the pattern collapse was evaluated using a test pattern provided by Kioxia: MoSi alloy-based attenuated phase shift material of 60-nm height, isolated pattern with minimum size of $50 \mathrm{~nm} \times 75 \mathrm{~nm}$.

Figure 10 shows the dependence of the PRE and the number of the pattern collapses on the freezing time. The water phase transitioned into solid at 80 and $120 \mathrm{~s}$ without crushing, and into solid with crushing at 180 and $600 \mathrm{~s}$. In this experiment, the ice surface was monitored, and the crushing of ice occurred at $\sim 150 \mathrm{~s}$ after the freezing step began. In addition, the PREs were $81 \%$ and $99 \%$ at 120 and $180 \mathrm{~s}$, respectively. The numbers of pattern collapses were 1 point of the

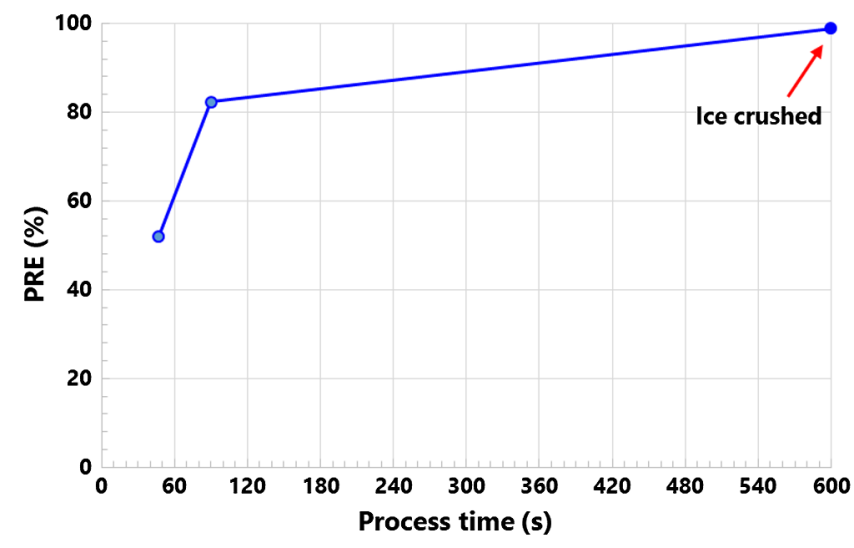

Fig. 9 PRE with respect to the freezing time. 


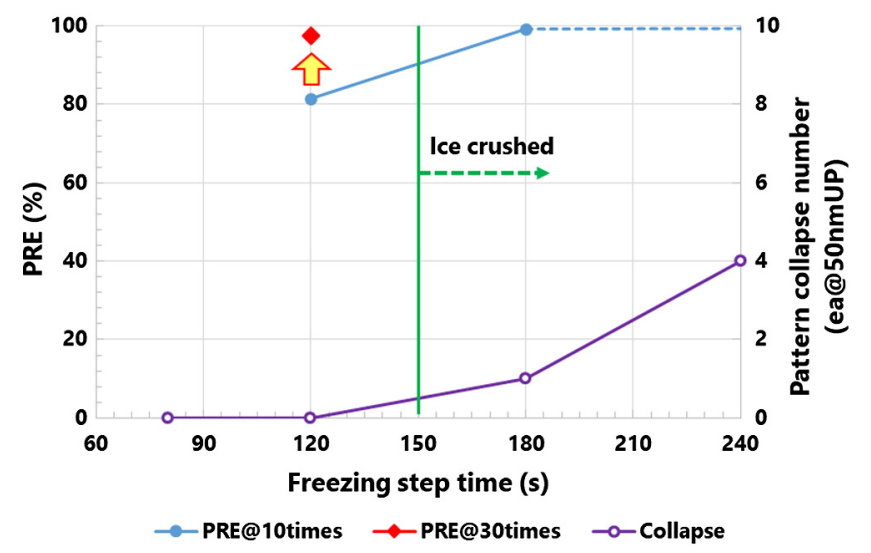

Fig. 10 PRE and the number of pattern collapses with respect to the freezing time.

pattern measuring $50 \mathrm{~nm}$ at $180 \mathrm{~s}$ and 4 points of the patterns measuring 50 and $55 \mathrm{~nm}$ at $240 \mathrm{~s}$. Thus, this indicates that the freezing time should be $<150 \mathrm{~s}$ to prevent the pattern collapse.

In this study, the details of the pattern collapse were not investigated. But considering that the test pattern is composed of MoSi alloy monolayer film, the collapse might be occurred at the interface between the substrate and the MoSi alloy.

To improve the PRE, 30 sets of freezing for $120 \mathrm{~s}$ and thawing steps were performed. Consequently, the PRE was improved to $98 \%$. By further investigation, the collapse of patterns with sizes equal to or smaller than $60 \mathrm{~nm}$ was observed in the ice crushing process. The proposed process can clearly distinguish the particles and patterns. In this case, the removal of the particles and the fine patterns was significantly different from that of the proposed mechanisms. The particles and the fine patterns are removed with the ice from the substrate when it is crushed. This phenomenon is considered to be understood the thawing starts after the ice crushed in Fig. 4: Fig. 4(e) $\rightarrow$ ice is crushed $\rightarrow$ thawing (rinsing). The entire water freezes Fig. 11(a), and continuous cooling causes the ice to shrink, eventually crushing along with particles and fine patterns Fig. 11(b). Given that $\mathrm{PRE}_{1}$ was estimated to be $\sim 7 \%$ (Sec. 4.1), many particles should be retained after one treatment cycle.

Now we discuss the consistency of the results in Fig. 10 and our model proposed with Fig. 11. The number of the pattern collapse seems to increase by prolonging freezing step time. And the number of crack on the ice also increases by prolonging freezing step time, but its number is much larger by a few orders than that of pattern collapse. So we speculate these phenomena as follows: adhesion force between the substrate and the MoSi alloy is much stronger than the peeling force when the ice crushed. So the peeling of the MoSi alloy pattern occurs stochastically. Therefore, the number of the pattern collapse increases slowly. This means that even in the ice crushed procedure, it is still a very soft treatment compare to the convenience treatment.

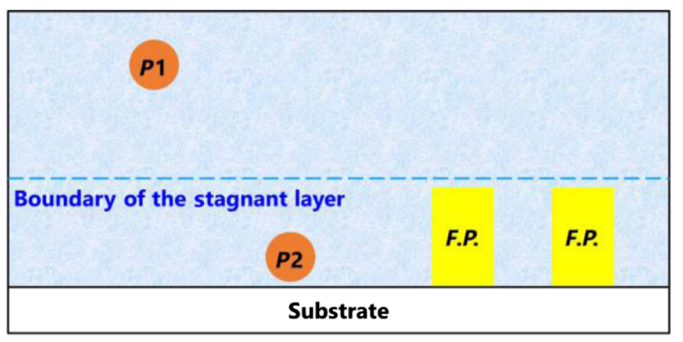

(a)

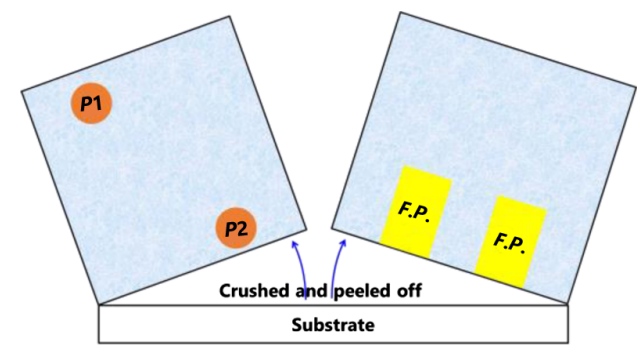

(b)

Fig. 11 Model of the long freezing duration and the subsequent ice crushing. (a) After the entire water is frozen [same as Fig. 4(e)] and (b) additional cooling causes the ice crush along with everything peeling from the substrate. 
Based on the above consideration, freezing should be completed prior to ice crushing to realize cleaning with high PRE and low-damage probability of fine patterns.

\subsection{Discussion on the Applicability and Limitation of the Freeze-Cleaning Method}

As above-mentioned, we discussed the model and results employing the tool designed and manufactured to realize the model. Although our proposed model is conceptual, data were limited: used only 80-nm-diameter sphere polystyrene particles on the blanket quartz for freezecleaning evaluation and only Att-PSM (MoSi alloy base material) with $50 \mathrm{~nm} \times 75 \mathrm{~nm}$ used for pattern collapse evaluation.

Here, we will discuss the applicability and the limitation of freeze-cleaning method.

First, we discuss the applicability to the other objects:

In our model, water infiltration into the gap between the particle and the substrate is the most important phenomenon to obtain the removal force of adhered particle from the substrate. As the above-mentioned discussion, if the gap between the particle and the substrate is submicron order, water tends to infiltrate to the gap by capillary force. But if the surface free energy of the particle and/or the surface of the substrate is too high to allow the water to infiltrate into the gap, removal force is not obtained. So the surface free energy of not only the particle and but also the surface of the substrate affects the applicability of the freeze-clean method. Surface free energy is varied by not only the material but also its surface condition.

If the gap itself is very narrow, water cannot infiltrate. If the particle has film shape and adheres on the substrate, the gap should be very narrow. So the particle shape also affects the applicability of the freeze-cleaning method.

Second, we discuss for heavy and/ or large particle removal:

In accordance with the above-mentioned discussion, we estimate some of the value, in case of the particle is $\operatorname{SiN}\left(\rho_{p}=3.44 \mathrm{~g} / \mathrm{cm}^{3}\right)$ of $80 \mathrm{~nm}$ in diameter. In this case, enough radius corresponded to Eq. (15) is estimated as

$$
r_{\mathrm{SiN}} \cong 288 \mathrm{~nm} \text {. }
$$

Equation (21) shows that if the FC particle size is larger than this number, particle weight can be ignored. Therefore if the FC particle size is $3.5 \mu \mathrm{m}$ in diameter, its terminal velocity and time of travel of $100 \mathrm{~nm}$ are regarded as the same value as Eqs. (16) and (18). In our system, the entire ice is frozen in some seconds, so FC particle with the diameter larger than $3.5 \mu \mathrm{m}$ is likely formed in every cycles and escapes from the stagnant layer. From this consideration it is thought even if the particle density is high, it will easily escape from the stagnant layer. And if the particle size is larger than $100 \mathrm{~nm}$, it means that the particle is larger than the stagnant layer, the particle can feel the drag force of Eq. (1) when rinsing. Then the particle may be removed from the surface along with the buoyancy and drag force in Eq. (1). Moreover when the ice freezing, the particle feels a volumetric expansion force: it acts as a removal force from the substrate. For the large particle, the gap between the particle and the substrate is also considered large. Then the amount of volume expansion is also considered large, and as a result, the removal force becomes large.

Finally, we discuss the heat shock during the treatment.

In our system, water is cooled from back side of the substrate. The surface temperature is saturated at $-70^{\circ} \mathrm{C}$ by supplying cryogenic $\mathrm{N}_{2}$ gas of $-100^{\circ} \mathrm{C}$ from the substrate back side. And during the thaw process, the surface temperature experiences between $-70^{\circ} \mathrm{C}$ to $20^{\circ} \mathrm{C}$. So the maximum temperature range is $12^{\circ} \mathrm{C}$. This temperature change may affect to the substrate properties. Especially, EUV blanks have very thin multilayer of Si and Mo, and also have absorbers via capping layer.

Currently, the criteria or limitations of the above-mentioned situation are not clear. Further evaluation should be needed. 


\section{Conclusions}

In this study, a technique for the removal of small-sized particles $(<100 \mathrm{~nm})$ from a substrate was developed and verified. Particles that were excessively small to be buried under the stagnant layer $(\sim 100 \mathrm{~nm})$ were removed from the substrate using the volumetric expansion force generated by the freezing of supercooled water, namely, rapid freezing, and escaped from the stagnant layer by buoyancy of the ice enclosing the particles that were grown by continuous cooling of the substrate. Slight change in volume, phase transition of half of the initial amount of water into ice, and distinction between the particle and the fine pattern upon freezing were particularly observed, followed by surface removal and the rise of the particle and the surrounding ice to the water surface by buoyancy. In the proposed freeze-cleaning process, the DIW on a substrate was cooled from below using cryogenic $\mathrm{N}_{2}$, and the DIW in the liquid-solid phase was then thawed. This technique was verified for the treatment of particles without affecting the fine patterns, thereby achieving a high PRE without pattern collapse. Moreover, given that DIW was used, no chemical discharges were observed. In our proposed model, discriminant method of patterns and particles is merely whether water can infiltrate into the gap between the substrate and the particle. At this point of view, we believe that proposed model can be widely applied to many situations, such as material and structure. In the same sense, there are some limitations of the proposed model. If the particles are in the form of a film and adhere tightly to the substrate, water cannot infiltrate into the gap. If the surface energy of the substrate and/or the particle is too high to allow water to infiltrate into the gap. In these cases, our proposed process cannot be applied. However, we believe that our proposed process can help the improvement in performance for removing the particles from the substrate with minimum affection to the substrate properties, by combining the current technologies, such as physical and/or chemical treatment. Because our proposed process uses only water and the temperature range from $-100^{\circ} \mathrm{C}$ to $20^{\circ} \mathrm{C}$. In these temperature ranges, it is considered that water does not heavily affect any chemical reaction.

The above-mentioned characteristic, namely, high PRE without pattern collapse or any chemical impact, has been recognized in this decade to be in a tradeoff relation.

The proposed technique has a potential to be employed to clean various masks, such as photomasks, EUV masks, and NIL templates, and can be applied to wafer processing.

Further investigation is required to consolidate and expand the applicability of the freezecleaning technique. We believe that the mechanism of lifting off particles from the substrate is commonly accepted; however, its limitations are not clear in terms of water infiltration to the gap, gap amount required, limitation to the material or its surface energy (contact angle), and so on. Given the unique characteristics of water with respect to its volumetric expansion upon freezing, it is required as the main material. However, several additives are suitable, which may improve the performance. Moreover, carbonated water is widely used as a rinsing liquid to prevent the degradation caused by the charge build-up on the substrate during the rinse process. Therefore, carbonated water was applied herein to freeze cleaning, and the results were highly similar. The saturation concentration of $\mathrm{CO}_{2}$ in water was $<100 \mathrm{ppm}$ at $20^{\circ} \mathrm{C}$; hence, the characteristics of water do not significantly vary.

In addition to several chemical treatment methods, conventional techniques, such as megasonic cleaning and two-fluid spray techniques, may be combined for freeze cleaning. Similarly, a long freezing duration of freeze cleaning, which induces ice crushing, may be applied under the following conditions: large pattern sizes, high adhesive strength, and no patterns (in the case of blanket cleaning).

\section{Acknowledgments}

The authors would like to acknowledge Hideaki Sakurai, Kosuke Takai, and Mana Tanabe from Kioxia Co., Ltd. for the sample provision, support, and helpful discussions. The authors would also like to thank Ivan Ganachev, Satoshi Nakamura, Minami Nakamura, Takumi Nagata, and Sadayuki Jimbo from Shibaura Mechatronics Corp. for their theoretical, technical support, and helpful discussions. This work was based on the presentation at SPIE Advanced Lithography $2020 .^{24}$ Finally, we would like to thank Editage for English language editing. To the best of our 
knowledge, the authors have no conflicts of interest, financial or otherwise. This study did not receive any external funding.

\section{References}

1. Y. Fukuzumi et al., "Optimal integration and characteristics of vertical array devices for ultra-high density, bit-cost scalable flash memory," in IEEE Int. Electron Dev. Meeting, pp. 449-452 (2007).

2. J. Jang et al., "Vertical cell array using TCAT (terabit cell array transistor) technology for ultra-high density NAND flash memory," in Proc. Symp. VLSI Technol., pp. 192-193 (2009).

3. M. Bohr, "The new era of scaling in a SoC world," in IEEE Int. Solid-State Circuits Conf-Dig. Tech. Papers (2009).

4. M. Drapeau et al., "Double patterning design split implementation and validation for the 32 nm node," Proc. SPIE 6521, 652109 (2007).

5. S. Natarajan et al., "A $14 \mathrm{~nm}$ logic technology featuring 2nd-generation FinFET, air-gapped interconnects, self-aligned double patterning and a $0.0588 \mu \mathrm{m}^{2}$ SRAM cell size," in IEEE Int. Electron Devices Meeting, pp. 3.7.1-3.7.3 (2014).

6. T. Hiraka et al., "UV-NIL templates for the $22 \mathrm{~nm}$ node and beyond," Proc. SPIE 6730, 67305P (2007).

7. I. Yoneda et al., "Study of nanoimprint lithography for applications toward $22 \mathrm{~nm}$ node CMOS devices," Proc. SPIE 6921, 692104 (2008).

8. T. Hattori, Ultra Clean Surface Processing of Silicon Wafers, Springer, Heidelberg/Berlin/ New York (1998).

9. T. Hattori, Development of Surface Contamination and Cleaning, Vol. 9, Elsevier, Amsterdam/New York (2017).

10. K. Dobashi et al., "Ultrafine particle removal using gas cluster ion beam technology," IEEE Trans. Semicond. Manuf. 26, 328-334 (2013).

11. B. G. Eynon, Jr. and B. Wu, Photomask Fabrication Technology, pp. 398-410, McGrawHill Companies, Inc., New York (2005).

12. H. Tomita, "Advanced semiconductor wafer cleaning technology," Chem. Times 3, 1-7, in Japanese (2009).

13. E. B. Moore and V. Molinero, "Structural transformation in supercooled water controls the crystallization rate of ice," Nature 479, 506-508 (2011).

14. H. Yokoyama, "Supercooled water and its structure," Yokohama City Univ. Inst. Repository Bull. Nat. Sci. 62, 11-34, in Japanese (2011).

15. J. R. Rumble, CRC Handbook of Chemistry and Physics, 99th ed., pp. 6-7, 6-12, Taylor \& Francis, London (2018).

16. R. J. Speedy, "Thermodynamic properties of supercooled water at 1 atm," J. Phys. Chem. 91, 3354-3358 (1987).

17. "Stokes' law," Wikipedia, https://en.wikipedia.org/wiki/Stokes\%27_law.

18. J. R. Rumble, CRC Handbook of Chemistry and Physics, 99th ed., 6-1, Taylor \& Francis, London (2018).

19. T. Sanada and M. Watanabe, "Physical fluid cleaning (3) jet, brush, and other topics," Chem. Times 4, 16-21, in Japanese (2015).

20. E. Martines et al., "Super hydrophobicity and super hydrophilicity of regular nanopatterns," Nano Lett. 5, 2097-2103 (2005).

21. T. Sanada et al., "Gas compression and dissolution in closed end tubes by applying pressure (liquid infiltration characteristics into fine structure by pressure)," Trans. JSME 82(838), 16-00048, in Japanese (2016).

22. M. Tanabe et al., "Novel cleaning technology for nanoparticle removal," Proc. SPIE 11148, $11148 \mathrm{~N}(2019)$.

23. M. Kamiya et al., "Substrate processing apparatus and substrate processing method," Japanese Patent No. 6738235 (2020).

24. D. Matsushima et al., "Unique freeze cleaning technology," Proc. SPIE 11326, 113260P (2020). 
Kei Hattori is a fellow at Shibaura Mechatronics Corporation and a visiting professor at Nagoya University. He had been engaged in R\&D and engineering for many kinds of wafer processing for 30 years in semiconductor device manufacture and currently is engaged in tool and process development for semiconductor device manufacturing. He is the inventor of more than 10 patents, author of four journal papers, and a member of the Japan Society of Applied Physics.

Daisuke Matsushima is a senior specialist at Shibaura Mechatronics Corporation. He is currently engaged in sales and had been engaged in photomask cleaning technology development for 15 years. He is the inventor of more than 10 patents.

Kensuke Demura is a senior specialist at Shibaura Mechatronics Corporation. He is engaged in photomask cleaning technology development for 10 years. He is the inventor of more than 10 patents.

Masaya Kamiya is an engineer at Shibaura Mechatronics Corporation. He is engaged in photomask cleaning technology development for five years. He is the inventor of more than 10 patents. 